\title{
Aplicaciones de los minerales arcillosos de Cayo Guan, Cuba, como adsorbentes de metales pesados y materia prima cerámica
}

\author{
D. FONSECA, F. BARBA, P. CALLEJAS, P. RECIO
}

Instituto de Cerámica y Vidrio - CSIC. Campus de Cantoblanco, c/ Kelsen 5, 28049 Madrid, España, flora@icv.csic.es

En Cayo Guan, municipio de Moa, hay junto a las importantes explotaciones industriales de lateritas, minerales arcillosos cuya caractarización química, mineralógica y evolución de fases con la temperatura han sido objeto de estudio por estos autores en un anterior trabajo; entre las características físicas, químicas y mineralógicas del yacimiento destacan los altos contenidos de óxidos de hierro y aluminio, bajas concentraciones de $\mathrm{SiO}_{2}$ y óxidos alcalinos, y la caolinita como mineral más abundante, además de hematita, gibsita y goethita en forma de geles.

Se pretende en esta investigación estudiar sus propiedades tecnológicas para dilucidar la posible aplicabilidad a estos recursos minerales en la fabricación de ladrillos, baldosas de pasta roja, gres rústico y para la inmovilización de metales pesados en efluentes acuosos.

El estudio por microscopía óptica de calefacción de los minerales arcillosos, indica que son unos materiales refractarios cuyo reblandecimiento aparece después de los $1500^{\circ} \mathrm{C}$. El diagrama de trabajabilidad de las diferentes arcillas y el cálculo de la plasticidad por el método de la cuchara de Casagrande presentan dentro de la zona favorable para la extrusión sólo a una de las muestras. Los diagramas de gresificación informan que es a partir de $1400{ }^{\circ} \mathrm{C}$ cuando se consigue una capacidad de absorción de agua $<0,6 \%$.

El diseño de un programa ha posibilitado el cálculo de múltiples composiciones de un gres porcelánico preparado a partir de dichas arcillas, modificadas con materias primas de bajo coste que facilitan la formación de fase vítrea (feldespato potásico y casco de vidrio) y/o incrementan el contenido de sílice (arena y diatomea utilizada como filtros en la industria cervecera). Se presentan los resultados de una de ellas (60\% de mineral arcilloso, $30 \%$ de feldespato potásico y $10 \%$ de diatomea) que calcinada a $1250{ }^{\circ} \mathrm{C}$ con una velocidad de calentamiento de $15{ }^{\circ} \mathrm{C} /$ min dio lugar a una absorción de agua de $0,8 \%$ y contracción lineal de $21 \%$ sin observarse deformación.

Por último, para estudiar su aplicación como adsorbente de metales pesados $\left(\mathrm{Cd}^{2+}, \mathrm{Cr}^{3+}\right)$, los minerales arcillosos se sometieron a un tratamiento ácido para eliminar su alto contenido en hierro y se compararon los resultados de la inmovilización de estos iones con los obtenidos con un reconocido material adsorbente como la vermiculita activada a 800 ${ }^{\circ} \mathrm{C}$, observándose que los minerales sometidos al tratamiento de lixiviación presentan una adsorción, tanto de $\mathrm{Cd}^{2+}$ como de $\mathrm{Cr}^{3+}$, por debajo de la conseguida con la vermiculita, sin embargo los que no se han sometido a dicho tratamiento presentan buen comportamiento frente al cromo; esto se debe a que estos minerales arcillosos no contienen en su composición apenas iones intercambiables $\left(\mathrm{Ca}^{2+}, \mathrm{Mg}^{2+}, \mathrm{Na}^{+}, \mathrm{K}^{+}\right)$, y aunque se hayan activado/lixiviado químicamente sólo la presencia de iones Fe es la que produce enlaces del tipo $\left(\mathrm{Cr}_{x} \cdot \mathrm{Fe}_{1-\mathrm{x}}\right)(\mathrm{OH})_{3}$ que favorecen la adsorción del $\mathrm{Cr}$.

Palabras clave: minerales arcillosos, adsorción de metales pesados, gres porcelánico, materia prima cerámica

\section{Application of clay minerals from Cayo Guan, Cuba, as sorbents of heavy metals and ceramic raw materials}

It has been studied by Analysis Heating Microscope - Optical the behaviour of some kaolinitic clays from a reservoir of Cayo Guan rich in iron oxides and low silica content proving to be a refractory materials whose softening appears after $1500{ }^{\circ} \mathrm{C}$. It has obtained the workability diagram of the different clay minerals calculating the plasticity by the method of Casagrande spoon; only one of the samples is in the area suitable for extrusion. Vitrification diagrams report that the capacity of water absorption is $<0.6 \%$ when the temperature of $1400{ }^{\circ} \mathrm{C}$ is achieved.

We have designed a program to calculate compositions of porcelain stoneware prepared from these modified clays adding low-cost raw materials that facilitate the formation of glassy phase ((potassium feldspar and glass cullet) and/or increase the silica (sand and diatomaceous earth used as filters in the brewing industry). With one of these compositions, prepared in the laboratory ( $60 \%$ of clay, $30 \%$ feldspar and $10 \%$ of diatomaceous earth), calcined at $1250{ }^{\circ} \mathrm{C}$ with a heating rate of $15{ }^{\circ} \mathrm{C} / \mathrm{min}$, the results were: water absorption $0.8 \%$, and linear shrinkage $21 \%$ without any deformation observed.

These clays have been treated with acid to eliminate its high iron content and study its application as an sorbent of heavy metals as $\mathrm{Cd}^{2+}, \mathrm{Cr}^{3+}$. The results of the immobilization of these elements have been compared with those obtained with thermally activated vermiculite at $800^{\circ} \mathrm{C}$, showing that the treated samples show sorption of both cadmium and chromium below the vermiculite, but the non-treated ones are suitable to remove chromium; this is because these clays do not contain in its composition exchangeable ions $\left(\mathrm{Ca}^{2+}, \mathrm{Mg}^{2+}, \mathrm{Na}{ }^{+}, \mathrm{K}{ }^{+}\right)$, and even if they are chemically activated only the presence of Fe ions is which produces form bindings $\left(\mathrm{Cr}_{x} \cdot \mathrm{Fe}_{1-x}\right)(\mathrm{OH})_{3}$ which favor $\mathrm{Cr}$ sorption. 


\section{INTRODUCCIÓN}

Las arcillas de la zona de Cayo Guan, municipio de Moa, conjuntamente con las lateritas, constituyen parte esencial de los suelos de la región nororiental de Cuba como productos finales de la meteorización de los gabros existentes en las márgenes de los ríos de la zona. Se pretende dar aplicabilidad a estos recursos minerales.

Teniendo en cuenta que la explotación industrial de dichas lateritas, fuente fundamental de la obtención de $\mathrm{Ni}$ y $\mathrm{Co}$, genera toneladas de residuos en forma de lodos con altos contenidos de hierro (entre 55 y $68 \%$ en peso) y otros óxidos metálicos (1), una posibilidad de uso racional podría ser que ambos, lodos y arcillas, se utilizaran como materias primas para preparar ladrillos acumuladores de calor (2). Otra aplicación sería su empleo en la fabricación de ladrillos para pantallas protectoras de radiaciones gamma debido a su alto contenido en hierro (hematita y magnetita) (3) ya que se ha comprobado la eficacia del empleo de ladrillos de ferromanganeso a pesar de su menor masa protectora frente al uso tradicional del cemento (4).

Estas arcillas han sido caracterizadas en un trabajo anterior (5). La presente investigación centra su objetivo, como una primera etapa, en conocer la posibilidad de orientarlas, en correspondencia con su comportamiento térmico, propiedades tecnológicas y contenido de hierro, en la fabricación de baldosas de pasta roja, tales como revestimientos porosos, gres porcelánico y gres rústico y estudiar su utilización en estado natural o activadas químicamente, para la inmovilización de metales pesados como el cadmio y el cromo en aguas residuales.

\section{PARTE EXPERIMENTAL}

\subsection{Muestras}

En este trabajo se han empleado las cinco muestras de un corte (6) realizado en la región de Cayo Guan, Moa (Fig. 1 a y b) ya caracterizadas físico-químicamente en un trabajo anterior (5). En la Tabla I se presenta a modo de resumen su composición química y mineralógica. Recordar también que la distribución acumulativa por tamaño de partículas en las
TABLA I.- COMPOSICIÓN QUÍMICA (\% EN PESO) Y MINERALÓGICA DE LAS MUESTRAS (5).

\begin{tabular}{|c|c|c|c|c|c|}
\hline Muestra & CG-1 & CG-2 & CG-3 & CG-4 & CG-5 \\
\hline $\mathrm{SiO}_{2}$ & 15,1 & 28,1 & 17,8 & 8,80 & 38,5 \\
\hline $\mathrm{Al}_{2} \mathrm{O}_{3}$ & 41,1 & 36,6 & 37,3 & 44,5 & 33,2 \\
\hline $\mathrm{TiO}_{2}$ & 0,90 & 0,37 & 0,32 & 0,39 & 0,34 \\
\hline $\mathrm{Fe}_{2} \mathrm{O}_{3}$ & 16,2 & 16,1 & 20,8 & 17,1 & 11,9 \\
\hline $\mathrm{MnO}$ & 1,23 & 0,08 & 0,08 & 0,02 & 0,03 \\
\hline $\mathrm{MgO}$ & 0,10 & 0,13 & 0,14 & 0,06 & 0,46 \\
\hline $\mathrm{CaO}$ & 0,06 & 0,05 & 0,26 & 0,06 & 0,04 \\
\hline $\mathbf{N a}{ }_{2} \mathrm{O}$ & 0,06 & 0,07 & 0,09 & 0,04 & 0,08 \\
\hline $\mathrm{K}_{2} \mathrm{O}$ & 0,11 & 0,02 & 0,03 & 0,01 & 0,01 \\
\hline PPC (\%) & 23,9 & 17,4 & 20,58 & 25,9 & 13,82 \\
\hline $\begin{array}{l}\text { Composición mineralógica: Fase mayoritaria caolinita, seguida de hematita, } \\
\text { gibsita, goethita, geles de aluminio y hierro, y cuarzo }\end{array}$ \\
\hline
\end{tabular}

cinco muestras, es típicamente bimodal, separados ambos modos por una fracción entre 125 y $45 \mu \mathrm{m}$ que siempre es menor que el 10\%. El mayor contenido en la fracción fina corresponde a las muestras CG-2 y CG-5, con contenidos superiores al $82 \%$ en partículas de tamaño $<45 \mu \mathrm{m}$ y menos del $8 \%$ de residuo a $125 \mu \mathrm{m}$. En CG-1, la capa más afectada por la meteorización, con alto grado de oxidación de los elementos fundamentalmente de hierro que se manifiestan en forma de nódulos difícilmente disgregables, el residuo mayor de $45 \mu \mathrm{m}$ corresponde con el $70 \%$ del material, mientras que la fracción $<45 \mu$ m supera ligeramente el 25\%. Las muestras CG-3 y CG-4 presentan granulometrías intermedias (5)

\subsection{Comportamiento térmico: Microscopía de calefacción y Dilatometría}

El estudio del comportamiento térmico del material arcilloso se llevó a cabo en un microscopio de calefacción (fotomicroscopio) Hesse Instrument, sobre probetas cilíndricas de $3 \mathrm{~mm}$ de diámetro y $2 \mathrm{~mm}$ de altura, obtenidas por compresión axial. El régimen de calentamiento seguido fue de $7^{\circ} \mathrm{C} / \mathrm{min}$.
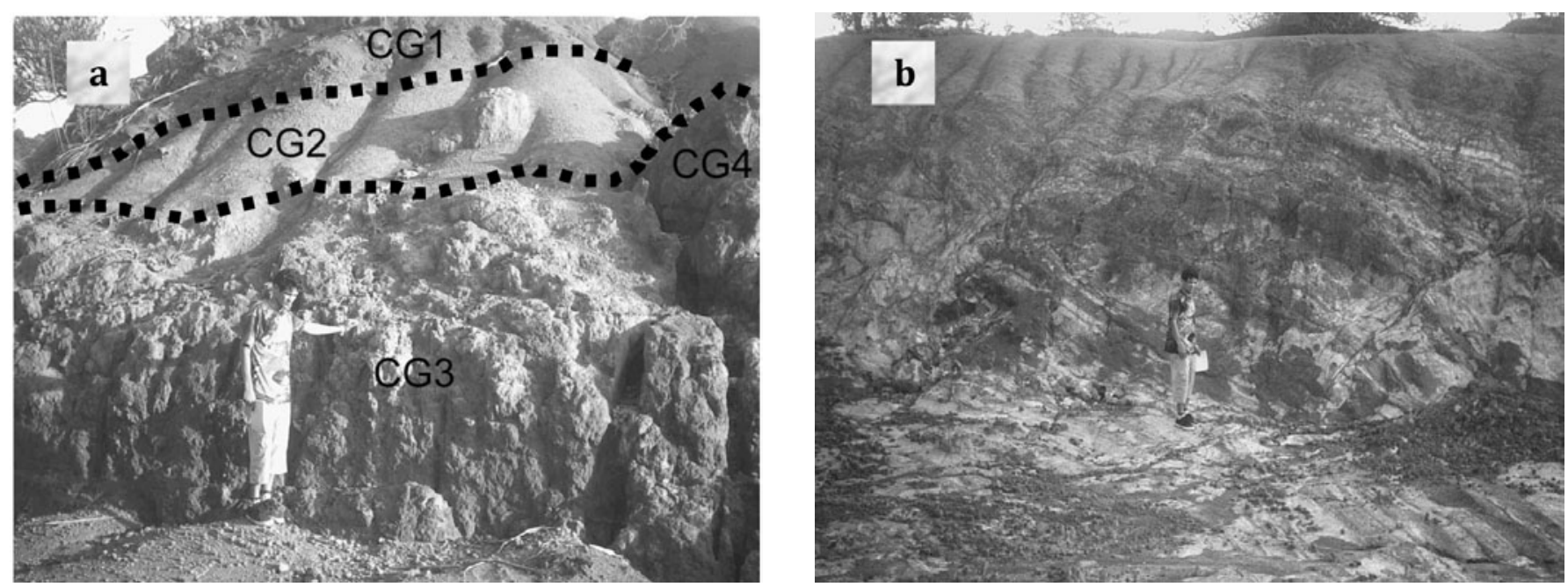

Fig. 1.- Vista panorámica del yacimiento y localización de las muestras a) CG-1 a CG-4; b) CG-5 
El estudio de sinterización dinámica de los materiales se realizó en un dilatómetro de la casa Netzch modelo 402 con un portamuestras de alúmina y en atmósfera oxidante. Se utilizaron probetas cilíndricas de $8 \mathrm{~mm}$ de longitud y $5 \mathrm{~mm}$ de diámetro, obtenidas por extrusión y prensado isostático (175 MPa). Todos los ensayos se llevaron a cabo en aire, en el intervalo $25-1200{ }^{\circ} \mathrm{C}$, con una velocidad de calentamiento y enfriamiento de $10^{\circ} \mathrm{C} / \mathrm{min}$.

\subsection{Propiedades tecnológicas}

El índice de plasticidad (Límites de Atterberg: límite líquido y plástico) de todas las muestras se realizó según las normas UNE, utilizando la "cuchara de Casagrande" para el límite líquido (7) y para el plástico, con la arcilla amasada en forma de cilindros de unos $3 \mathrm{~mm}$ de diámetro, calculando la pérdida de agua sufrida en el momento en que éstos comienzan a agrietarse sobre un molde de escayola (8).

La determinación de la absorción de agua y la contracción lineal para obtener los diagramas de gresificación se realizaron con probetas de $5 \mathrm{~g}$ de muestra de tamaño de partícula $<125$ $\mu \mathrm{m}$ y con $5 \%$ de humedad, obtenidas por prensado uniaxial (16 MPa). Se promediaron dos valores para cinco temperaturas de calcinación: $1100,1200,1300,1400$, y $1500{ }^{\circ} \mathrm{C}$, siguiendo un ciclo de calentamiento de $10{ }^{\circ} \mathrm{C} / \mathrm{min}$ y estancia en el horno a la temperatura final de $2 \mathrm{~h}$ (9)

La absorción de agua (A.A) se realizó siguiendo la norma UNE 67-027-84 para ladrillos de arcilla cocida (10), introduciendo la pieza calcinada en agua hirviendo durante dos horas.
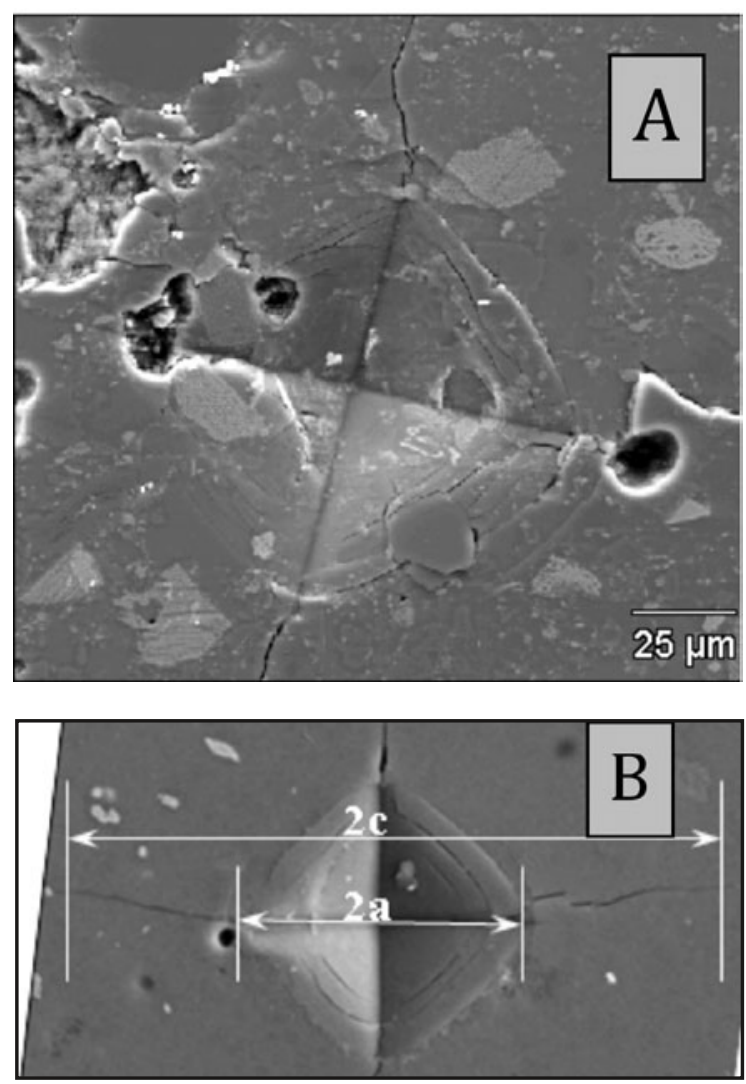

Fig. 2.- A) Huella obtenida con el indentador de Vickers B) Medidas de a y
Para la determinación de la contracción lineal (C.L) se utilizó la norma UNE-EN ISO (11) y la expresión :

$$
C . L(\%)=\frac{L_{i}-L_{f}}{L_{i}} 100
$$

donde $L_{i}$ es la longitud inicial de la pieza cruda y $L_{f}$ la longitud final (después de calcinada).

\subsection{Diseño de composiciones de gres. Propiedades mecánicas}

Fijando la composición de un gres patrón y utilizando la herramienta SOLVER de Microsoft Excell, se han calculado posibles composiciones de mezclas de estas arcillas con materias primas de bajo coste como modificadores.

Con las composiciones calculadas se prepararon, por prensado uniaxial (16 MPa), probetas de $2,5 \mathrm{~g}$ y $20 \mathrm{~mm}$ de diámetro que se sometieron a un ciclo de cocción de $15{ }^{\circ} \mathrm{C} /$ min hasta $1250{ }^{\circ} \mathrm{C} / 90 \mathrm{~min}$.

Las propiedades mecánicas (microdureza y tenacidad) del gres obtenido se determinaron siguiendo la norma ASTM (12) para cerámicas avanzadas; se utilizó el indentador Vickers aplicando una carga de $5 \mathrm{kgf}$ durante 10s; se midió la longitud de la grieta más larga (2c) y la diagonal de la huella (2a) (Fig. 2). Se realizaron los cálculos según las ecuaciones [2] y [3]:

$$
H v=\frac{0,464 \cdot P}{a^{2}}
$$

donde: $\mathrm{P}$ es la carga aplicada en $\mathrm{kgf}$; a: longitud de la diagonal $(\mu \mathrm{m})$

La tenacidad $\mathrm{K}_{\mathrm{IC}}$ se determinó por la ecuación [3], donde Hv está expresada ahora en GPa para obtener $\mathrm{K}_{\mathrm{IC}}$ en MPa.m ${ }^{1 / 2}$ :

$$
K_{I C}=0,16 H_{v}^{0,5}\left(\frac{c}{a}\right)^{-1,5}
$$

No se ha realizado la resistencia a la abrasión, que queda pendiente.

\subsection{Capacidad de adsorción de iones de metales pesados}

Para el estudio de la capacidad de adsorción (S) de los metales $\mathrm{Cr}^{3+}$ y $\mathrm{Cd}^{2+}$ se ha puesto en contacto $1 \mathrm{~g}$ de muestra (tamaño de partícula $<60 \mu \mathrm{m}$ ) con $50 \mathrm{ml}$ de diferentes

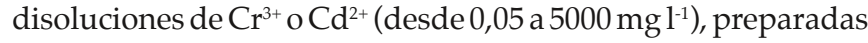
en medio $\mathrm{HNO}_{3} 0,01 \mathrm{~N}(\mathrm{pH}=4)$ a partir de los cloruros de los correspondientes cationes. Se mantuvo constante la relación adsorbato/adsorbente $(1 / 10)$ y el tiempo de agitación, que fue de $20 \mathrm{~h}$ para asegurar que el sistema alcanzara el equilibrio. En el filtrado del sistema fueron analizados ambos cationes por espectrometría de emisión atómica de acoplamiento inductivo (ICP-OES) para conocer la adsorción, y por diferencia, la retención $(\mathrm{R})$ utilizando las ecuaciones [4] y [5].

$$
\begin{aligned}
& \text { Retención (\%): } R=\left[1-\left(\frac{C e q}{C o}\right)\right] * 100 \\
& \text { Sorción }(\mathrm{mg} / \mathrm{kg}): \quad S=\frac{(\text { Co }-C e q) * V}{m}
\end{aligned}
$$


donde Co y Ceq son la concentración inicial y final, respectivamente en $\mathrm{mg} \mathrm{l}^{-1} ; V$, volumen de disolución en $\mathrm{ml}$ y $m$, masa del material en gramos.

\section{RESULTADOS Y DISCUSIÓN}

\subsection{Comportamiento térmico de los minerales.}

Los cambios volumétricos de las muestras obtenidas por extrusión y prensado isostático se observan en la Fig. 3 a y b. La curva dilatométrica muestra tres tramos bien definidos, $240-700{ }^{\circ} \mathrm{C}, 700-1120^{\circ} \mathrm{C}$ y $1120-1200{ }^{\circ} \mathrm{C}$, en los que se han estimado contracciones lineales de $2,85 \% ; 6,98 \%$ y 18,2 \% respectivamente. La contracción a temperaturas inferiores a $240{ }^{\circ} \mathrm{C}$ es apenas perceptible.

En el intervalo de temperaturas estudiado, los tres componentes cristalinos principales, caolinita, $\mathrm{Al}_{2} \mathrm{Si}_{2} \mathrm{O}_{5}(\mathrm{OH})_{4^{\prime}}$ gibsita, $\mathrm{Al}(\mathrm{OH})_{3}$ y goethita, $\alpha-\mathrm{FeOOH}$, sufren procesos de deshidroxilación, deshidratación y descomposición de acuerdo con las siguientes reacciones:
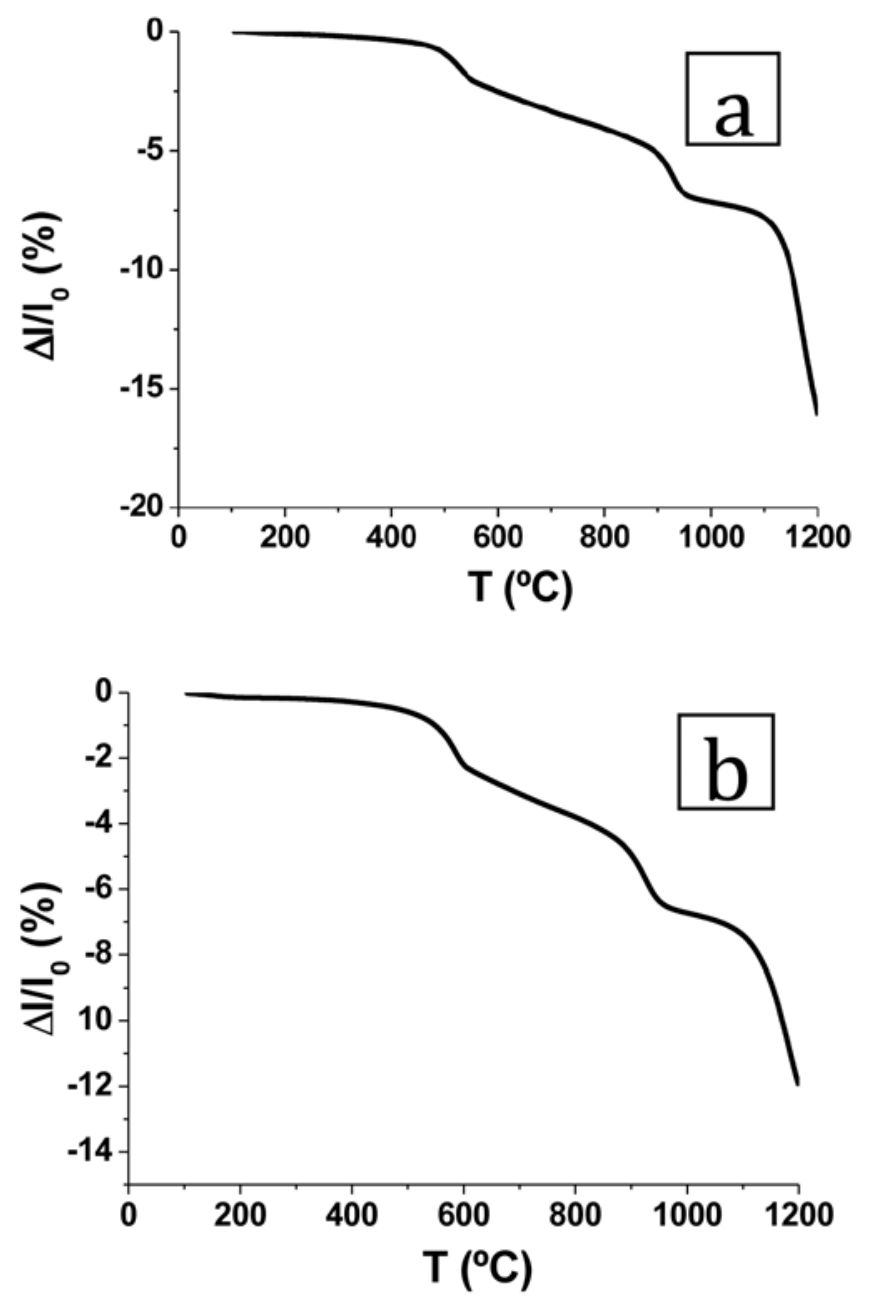

Fig. 3- Curvas dilatométrica de la muestra CG-5 a) por extrusión b) por prensado isostático

$$
\begin{aligned}
& \mathrm{Al}_{2} \mathrm{Si}_{2} \mathrm{O}_{5}(\mathrm{OH})_{4} \rightarrow \mathrm{Al}_{2} \mathrm{Si}_{2} \mathrm{O}_{7}+2 \mathrm{H}_{2} \mathrm{O} \rightarrow 1 / 33 \mathrm{Al}_{2} \mathrm{O}_{3} \cdot 2 \mathrm{SiO}_{2} \\
& 2 \mathrm{Al}(\mathrm{OH})_{3} \rightarrow \chi-\mathrm{Al}_{2} \mathrm{O}_{3}+3 \mathrm{H}_{2} \mathrm{O} \rightarrow \kappa-\mathrm{Al}_{2} \mathrm{O}_{3} \rightarrow \alpha-\mathrm{Al}_{2} \mathrm{O}_{3}(13) \\
& 2 \alpha-\mathrm{FeOOH} \rightarrow \alpha-\mathrm{Fe}_{2} \mathrm{O}_{3}+\mathrm{H}_{2} \mathrm{O}(14)
\end{aligned}
$$

Sin embargo el hábito de la curva es similar al observado en arcillas caoliníticas casi puras (15), lo que indica que la transformación, entre 450 y $550{ }^{\circ} \mathrm{C}$, de caolinita en la forma deshidroxilada, metacaolinita, y su posterior transformación a alta temperatura, en mullita, $3 \mathrm{Al}_{2} \mathrm{O}_{3} .2 \mathrm{SiO}_{2^{\prime}}$ son los principales responsables de la contracción de esos materiales arcillosos. Temuujin (16) ha comprobado que en mezclas de gibsita y caolinita, la secuencia de transformación seguida por ambas materias primas es similar a la de las especies individuales y que metacaolinita y una alúmina transitoria eran los constituyentes mayoritarios incluso a altas temperaturas. Solo a partir de $1100{ }^{\circ} \mathrm{C}$ detectaban pequeñas cantidades de $\alpha-\mathrm{Al}_{2} \mathrm{O}_{3}$ junto a la mullita primaria procedente de la transformación de la caolinita. La deshidratación de gibsita no presenta contracción en el intervalo de temperaturas 240-700 ${ }^{\circ} \mathrm{C}$ (13) y, aunque la transformación de goethita hematita significa un decrecimiento en el parámetro $c$ y un incremento en el parámetro $b$ de la celdilla unidad de goethita (14), la deshidratación del oxihidróxido de hierro no parece modificar la curva dilatométrica. Paralelamente los cristales de hematita resultantes de la deshidratación de goethita, muestran a partir de $900{ }^{\circ} \mathrm{C}$ efectos de sinterización, primero sus agujas empiezan a redondearse y finalmente los cristales individuales empiezan a crecer (17). Finalmente, entre 1020 y $1200{ }^{\circ} \mathrm{C}$, tercer escalón de la curva dilatométrica, tiene lugar la cristalización de cristobalita a partir de la sílice amorfa y el crecimiento de los cristales de mullita.

A $1200^{\circ} \mathrm{C}$, temperatura máxima del ensayo, la sinterización del material no ha finalizado.

Del análisis del comportamiento térmico mediante la microscopía de calefacción (Fig. 4) se deduce la alta refractariedad de estas arcillas con temperaturas de fusión por encima de $1500{ }^{\circ} \mathrm{C}$ (excepto CG-4), temperatura a la cual aún no se observan signos de cambio de estado físico a pesar de que la contracción volumétrica es elevada, lo que no significa que haya habido sinterización completa ni formación de fase líquida (gresificación) suficiente para lograr la máxima densificación, como se demostrará más adelante, sólo han tenido lugar procesos de deshidroxilación durante el calentamiento. A esta temperatura, $>1500{ }^{\circ} \mathrm{C}$, aún no se pueden definir los puntos característicos de reblandecimiento, fusión y fluidez, aunque se pudo comprobar que existen pequeñas difusiones de hierro, fundamentalmente en la

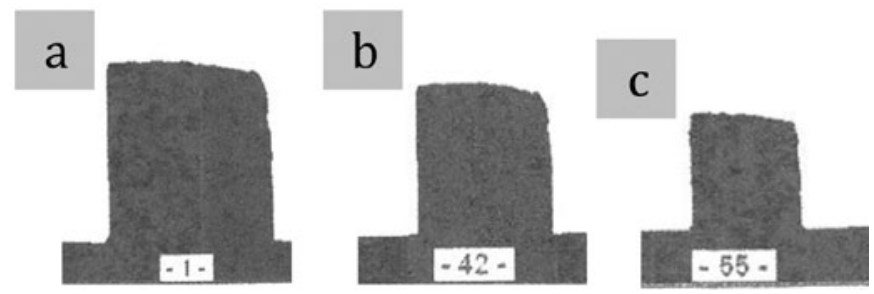

Fig. 4- Comportamiento térmico de CG-5 bajo microscopía de calefacción a a) $23^{\circ} \mathrm{C}$; b) $1200^{\circ} \mathrm{C}$ c) $1500^{\circ} \mathrm{C}$ 
TABLA II.- ÍNDICE DE PLASTICIDAD DE LAS MUESTRAS

\begin{tabular}{|c|c|c|c|}
\hline Muestra & $\begin{array}{c}\text { Lím Líquido } \\
(\mathbf{\%})\end{array}$ & $\begin{array}{c}\text { Lím plástico } \\
\mathbf{( \% )}\end{array}$ & $\begin{array}{c}\text { Índice de } \\
\text { plasticidad (\%) }\end{array}$ \\
\hline CG-1 & 34,16 & 27,90 & 6,26 \\
\hline CG-2 & 47,90 & 35,31 & 12,59 \\
\hline CG-3 & 39,46 & 33,92 & 5,54 \\
\hline CG-4 & 36,29 & 35,77 & 0,52 \\
\hline CG-5 & 47,37 & 42,60 & 4,77 \\
\hline
\end{tabular}

muestra CG-3, la cual se adhiere al portamuestras y CG-4, que prácticamente funde. En la CG-1 y CG-5 la difusión del hierro es menor, originando una huella de color rojo intenso.

\subsection{Evaluación de las propiedades tecnológicas de los minerales}

En la Tabla II se pueden observar los bajos índices de plasticidad obtenidos, $<13 \%$ en todos los casos, que podrían atribuirse a la gran cantidad de material no arcilloso, fundamentalmente óxidos de hierro y alúmina, altos porcentajes de partículas de gran tamaño, así como a la forma de éstas. Sólo la muestra CG-2, se ubica en el diagrama de Atterberg (Fig. 5) en la zona favorable para la extrusión (18). Las demás muestras requerirán para su procesamiento cerámico de aditivos (fundentes y desgrasantes, étc.) que les confieran propiedades aceptables (19).

En la Fig. 6 (a y b) se presentan los diagramas de gresificación (contracción lineal y absorción de agua) de CG-1 y CG-5. Los resultados de los ensayos de absorción de agua para todas las muestras a $1100{ }^{\circ} \mathrm{C}$ son demasiado elevados (23-35 \%) respecto a los esperados para cualquier material cerámico (2-21). Estos valores indican que las muestras estudiadas a esta temperatura, óptima para productos de gres y ya un poco elevada para ladrillos, no han alcanzado una buena densificación por lo que su porosidad abierta es elevada y da lugar al fácil desprendimiento de material durante su manipulación (baja resistencia mecánica). A 1200 ${ }^{\circ} \mathrm{C}$, la absorción aún sigue siendo muy alta (entre $12 \%$ y 33

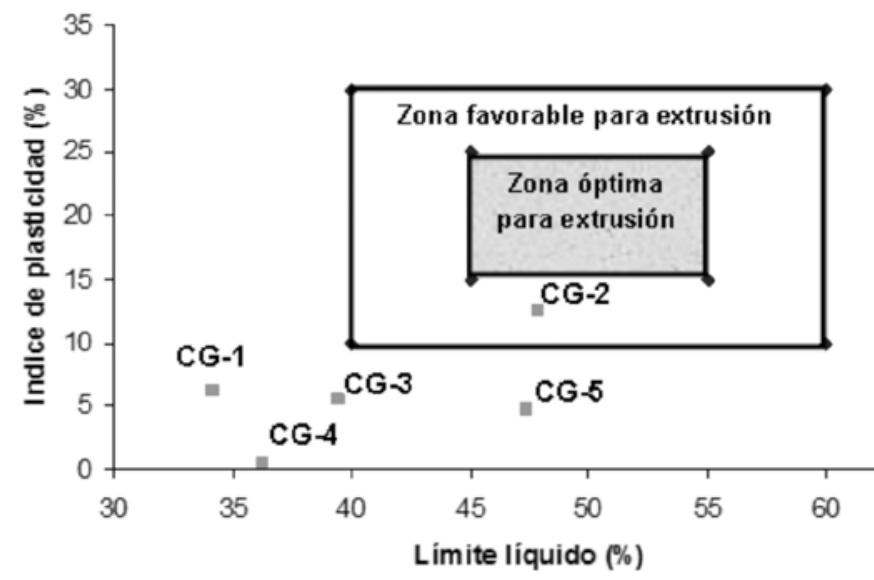

Fig. 5.- Ubicación de las muestras CG-1 a CG-5 en el Diagrama de Atterberg
$\%)$, fundamentalmente para las muestras CG-3 y CG-4. Al llegar a $1400{ }^{\circ} \mathrm{C}$ la absorción de agua se hace cero para las muestras CG-1, CG-2 y CG-5 cuyas probetas presentan un cierto brillo metálico, no siendo así para CG-3 y CG-4 que aún absorben agua en un $15,38 \%$ y $9,84 \%$ respectivamente. A $1500{ }^{\circ} \mathrm{C}$ la capacidad de absorción de agua es nula para todas las muestras.

La contracción lineal, que depende principalmente de la composición mineralógica y la compacidad alcanzada por la pieza moldeada, aumenta con la temperatura, debido a las pérdidas por calcinación que son altas en estos minerales, como ya se ha discutido, y a los procesos de transformación de fases que tienen lugar durante el calentamiento. A $1100{ }^{\circ} \mathrm{C}$ presentan contracciones entre el $6 \%$ y el $17 \%$ llegando a ser del orden del $40 \%$ para $1500{ }^{\circ} \mathrm{C}$.

Este comportamiento se debe a los bajos contenidos de sílice, alcalinos y otros desgrasantes que presentan las muestras, formadores de fase vítrea y los altos contenidos de alúmina: Los altos contenidos $\mathrm{de}_{\mathrm{Al}_{2} \mathrm{O}_{3}}$ (entre 38 y $45 \%$ en peso, CG-1 a CG-4, no así en CG-5), así como los bajos contenidos de $\mathrm{SiO}_{2}(<<40 \%$ en peso) en todas las muestras, no son los deseados para una arcilla cerámica. La combinación de bajos porcentajes de $\mathrm{SiO}_{2}$ y altos de $\mathrm{Al}_{2} \mathrm{O}_{3^{\prime}}$ asociados con altas pérdidas por calcinación, indican alta cantidad de filosilicatos y pequeñas de cuarzo (22). Además los contenidos
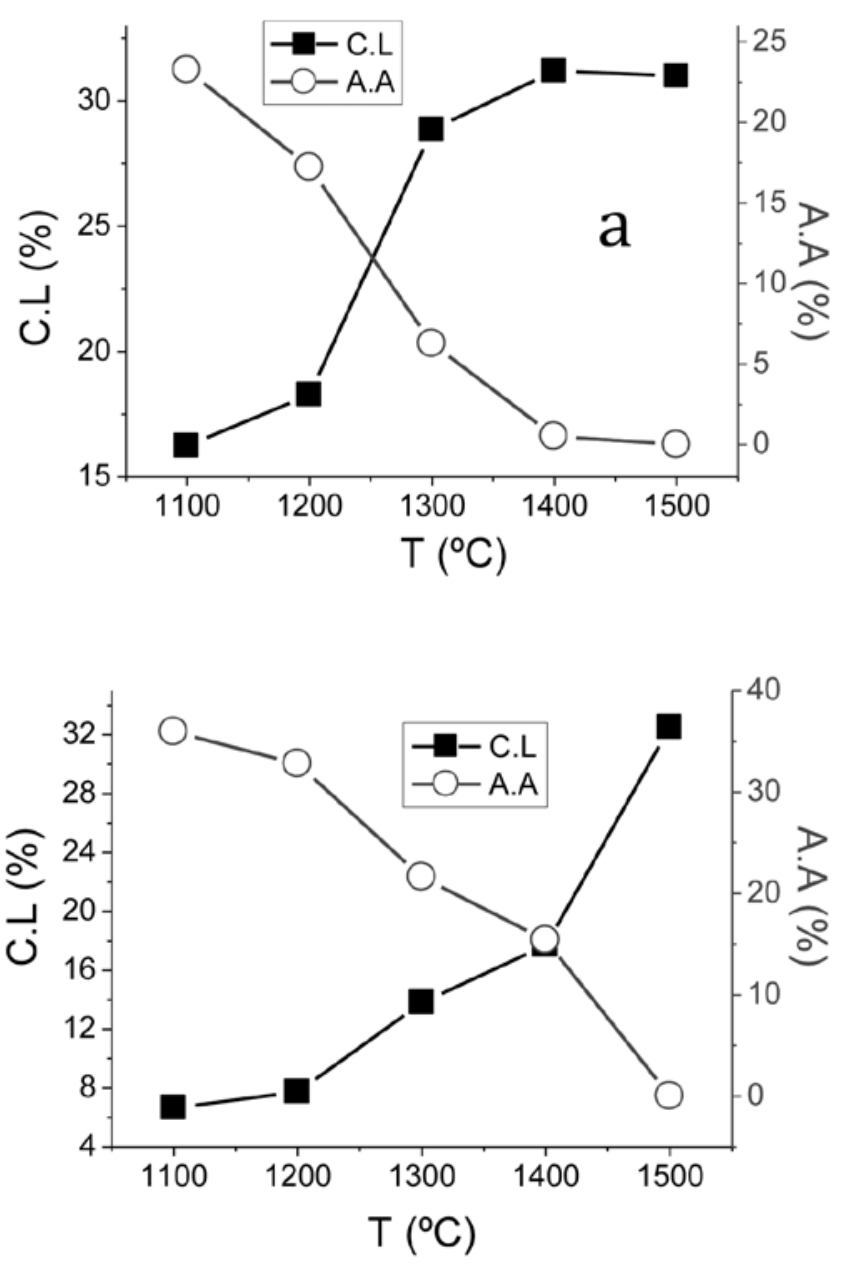

Fig. 6.- Diagrama de Gresificación de las arcillas a) CG-1, b) CG-5 
TABLA III.- COMPOSICIÓN QUÍMICA POR ICP-OES (\% EN PESO) DE LAS MATERIAS PRIMAS MODIFICADORAS PARA DISEÑAR GRES

\begin{tabular}{|l|c|c|c|c|c|c|c|c|c|}
\hline Materia prima & $\mathrm{SiO}_{2}$ & $\mathrm{Al}_{2} \mathrm{O}_{3}$ & $\mathrm{Fe}_{2} \mathrm{O}_{3}$ & $\mathrm{MgO}$ & $\mathrm{CaO}$ & $\mathrm{TiO}_{2}$ & $\mathbf{N a}_{2} \mathrm{O}$ & $\mathrm{K}_{2} \mathrm{O}$ & $\mathbf{P P C}$ \\
\hline Feldespato Potásico de Segovia & 68,7 & 16,6 & 0,1 & 0,1 & 0,5 & - & 2,6 & 11,0 & 0,60 \\
\hline Casco de vidrio & 72,5 & 1,3 & 0,17 & 0,42 & 11,8 & 0,24 & 12,8 & 0,81 & - \\
\hline
\end{tabular}

PPC: pérdida por calcinación a $1000^{\circ} \mathrm{C}$

de óxidos alcalinos (que actúan como formadores de fase líquida viscosa alrededor de $\operatorname{los} 700-800{ }^{\circ} \mathrm{C}$, contribuyendo al llenado de los poros y consecuentemente a incrementar la densificación) son bajos $(<0,5 \%$ en peso). La escasa densificación observada, podría deberse a la sinterización en estado sólido con predominio de transporte de masa, acompañada de crecimiento de grano (23).

A mayores temperaturas $\left(1400-1500{ }^{\circ} \mathrm{C}\right)$ ya se observa que ha habido un proceso importante de densificación dado por la disminución de la absorción de agua (0-2\%) y el incremento de la contracción lineal.

Para las arcillas CG-1 y CG-5 se observa la tendencia a existir un intervalo de temperaturas durante el cual ocurre la gresificación (Fig 6 a y b), no siendo así para CG-3 que logra su máxima densificación a la temperatura a partir de la cual el hierro se difunde produciendo la adhesión de la muestra al soporte.
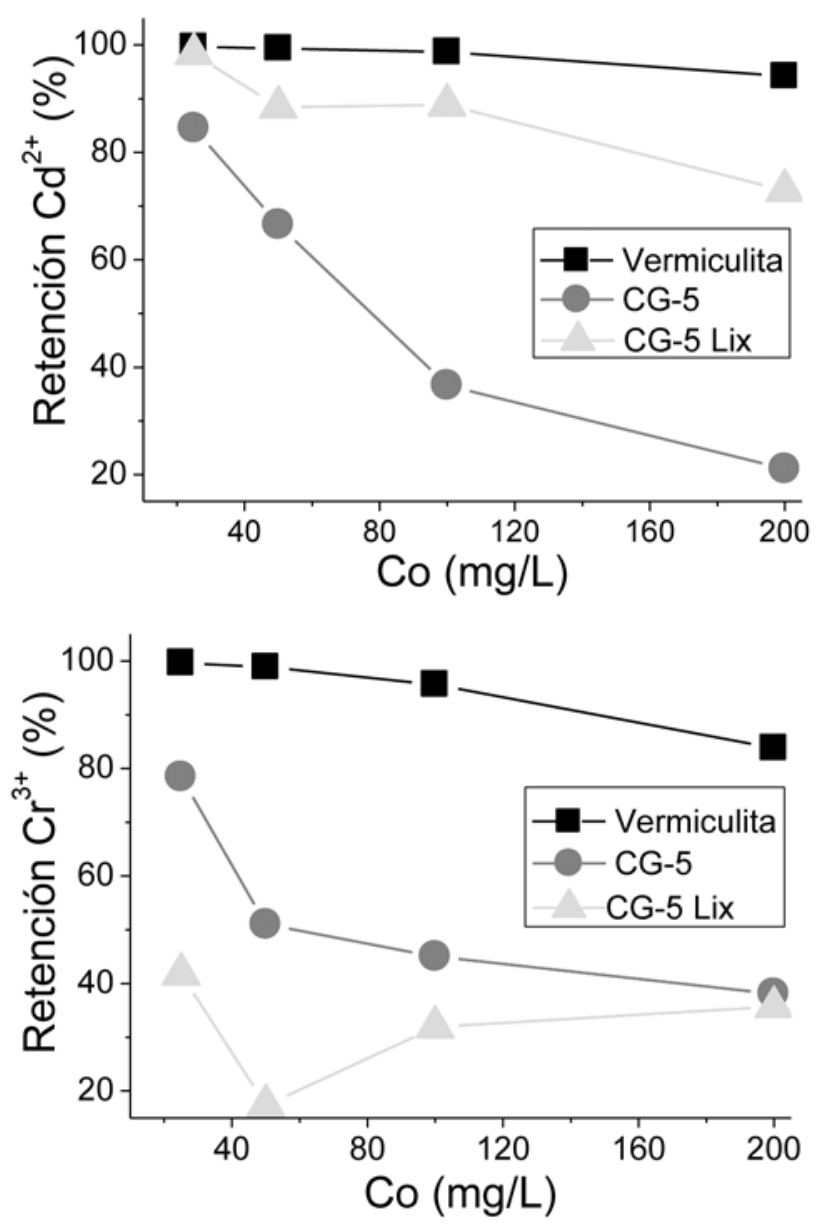

Fig. 7.- Retención de iones $\mathrm{Cd}$ y Cr

\subsection{Formulación de un gres y sus propiedades}

Para el diseño de las composiciones de gres porcelánicos se utilizaron tres parámetros:

1. Una composición química fija teórica de gres (\% en peso): 65 de $\mathrm{SiO}_{2}, 25 \mathrm{de} \mathrm{Al}_{2} \mathrm{O}_{3}+\mathrm{Fe}_{2} \mathrm{O}_{3}$ y 10 de $\mathrm{Na}_{2} \mathrm{O}+\mathrm{K}_{2} \mathrm{O}$

2. La composición química de las cinco muestras de arcilla objeto de estudio

3. La composición de las materias primas modificadoras para aportar sílice: a) una arena de 99,9\% de sílice, b) casco de vidrio; c) un feldespato potásico. (Tabla III

El programa informático da lugar a una amplia gama de mezclas posibles. En este trabajo, a modo de ejemplo, se discute la mezcla obtenida al fijar como materias primas de partida $60 \%$ de muestra CG-2; $30 \%$ del feldespato potásico
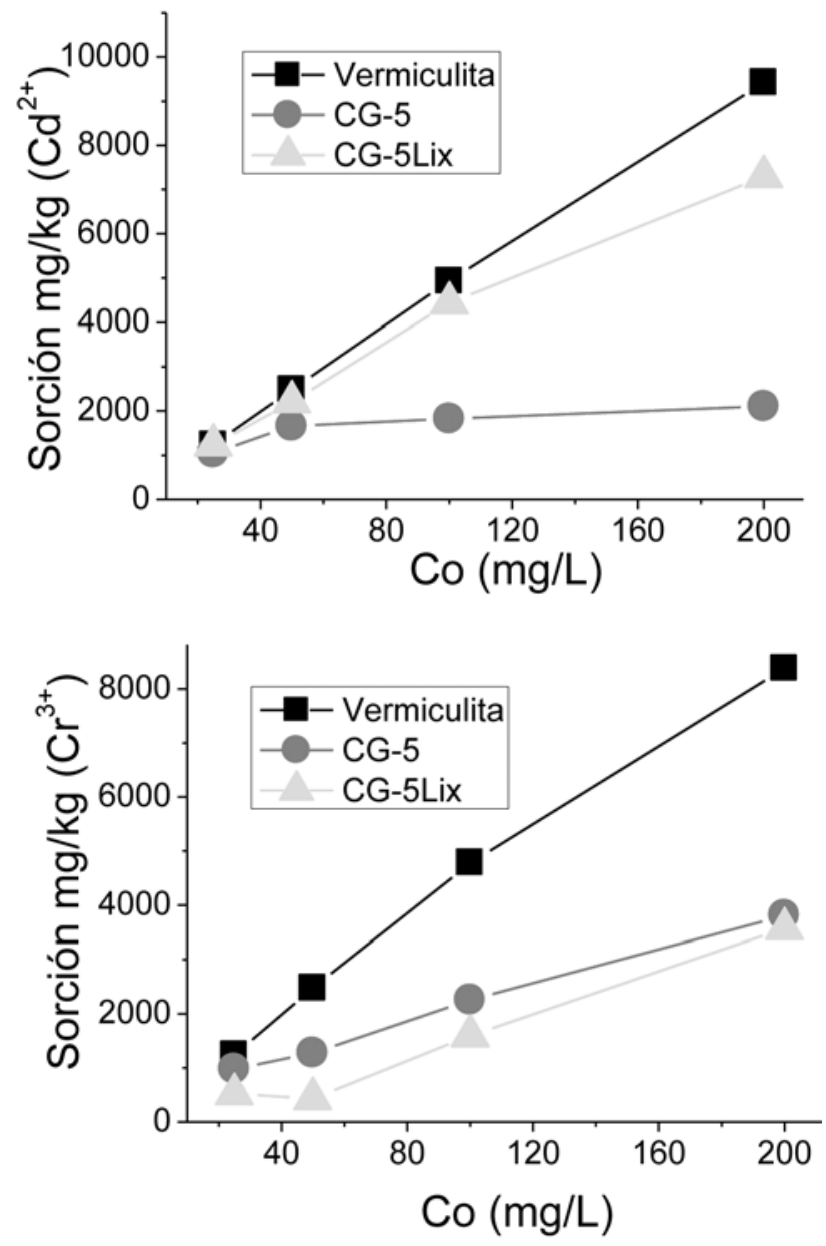

Fig. 8.- Adsorción de iones $\mathrm{Cd}$ y $\mathrm{Cr}$ 


\begin{tabular}{|c|c|c|c|c|c|c|c|c|}
\hline $\mathrm{SiO}_{2}$ & $\mathrm{Al}_{2} \mathrm{O}_{3}$ & $\mathrm{Fe}_{2} \mathrm{O}_{3}$ & $\mathrm{MgO}$ & $\mathrm{CaO}$ & $\mathrm{TiO}_{2}$ & $\mathrm{Na}_{2} \mathrm{O}$ & $\mathrm{K}_{2} \mathrm{O}$ & PPC \\
\hline 47,47 & 26,94 & 9,69 & 0,11 & 0,18 & 0,24 & 0,82 & 3,31 & 10,44 \\
\hline
\end{tabular}

PPC: pérdida por calcinación a $1000^{\circ} \mathrm{C}$

TABLA V.- ANÁLISIS QUíMICO (\% EN PESO) DE LA VERMICULITA

\begin{tabular}{|c|c|c|c|c|c|c|c|c|}
\hline $\mathrm{Al}_{2} \mathrm{O}_{3}$ & $\mathrm{CaO}$ & $\mathrm{Cr}_{2} \mathrm{O}_{3}$ & $\mathrm{Fe}_{2} \mathrm{O}_{3}$ & $\mathrm{MgO}$ & $\mathrm{SiO}_{2}$ & $\mathrm{Na}_{2} \mathrm{O}$ & $\mathbf{K}_{2} \mathrm{O}$ & $\mathbf{P P C}$ \\
\hline 9,30 & 0,72 & 0,02 & 6,72 & 19,7 & 45,86 & 0,05 & 4,00 & 11,0 \\
\hline
\end{tabular}

PPC: pérdida por calcinación a $1000^{\circ} \mathrm{C}$

y $10 \%$ de la arena, que conduce a la composición química teórica que se presenta en la Tabla IV

Con esta mezcla se prepararon unas probetas que se calcinaron (apartado 2.3). El material obtenido respecto del gres teórico es deficitario en sílice y alcalinos y sus propiedades tecnológicas: absorción de agua y contracción lineal para las probetas calcinadas a $1250{ }^{\circ} \mathrm{C}$ fueron de 0,28 y $21 \%$ respectivamente. El valor de absorción de agua es adecuado para un gres, según la bibliografía (24). La contracción lineal de $21 \%$ es alta, teniendo a su favor que no se aprecian grietas, fisuras, ni deformación. La temperatura de calcinación de $1250{ }^{\circ} \mathrm{C}$ se podría aceptar para emprender un proceso de producción a gran escala.

En la huella obtenida con el indentador Vickers se han medido los valores de a y c (Fig 2b), con las que se calcularon las propiedades mecánicas (Dureza Vicker: $\mathrm{Hv}=8,5 \mathrm{GPa}$; Tenacidad: $\left.\mathrm{K}_{\mathrm{IC}}=2,83 \mathrm{MPa} \cdot \mathrm{m}^{1 / 2}\right)$. En la figura se pueden observar las fases de cuarzo $\left(\mathrm{SiO}_{2}\right)$, corindón $\left(\alpha-\mathrm{Al}_{2} \mathrm{O}_{3}\right)$, compuestos de hierro y microcristales de mullita dispersos en la fase vítrea de este gres, tal como era de esperar por el estudio previo de la evolución de fases con la temperatura realizado de estas arcillas (5) y la presencia de los modificadores adicionados.

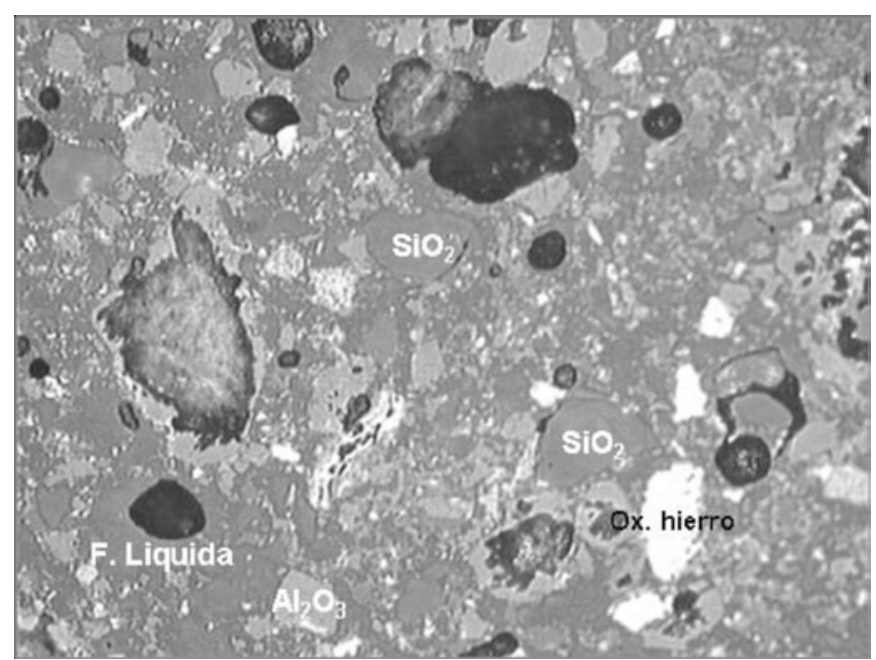

Fig .9 - Observación por microscopía óptica de reflexión del gres preparado con la arcilla CG- 2

\subsection{Capacidad de adsorción de metales pesados}

Debido a la presencia de una fase arcillosa considerable en las muestras y a sus altos contenidos de oxi-hidróxidos de hierro y aluminio (5), se estudió su capacidad para ser utilizadas como inmovilizadoras de metales pesados en efluentes acuosos. Como muestra de referencia se utilizó una vermiculita (Tabla V), cuya capacidad de absorción de metales pesados ha sido determinada en un trabajo previo (25)

Los ensayos de adsorción (apartado 2.5) se realizaron sobre muestra natural y tratada con $\mathrm{HCl}$ al $10 \%$, hasta ebullición, durante $2 \mathrm{~h}$, para eliminar sus geles de hierro y aluminio.

Los resultados de adsorción y retención para la muestra CG-5 se presentan en las Fig. 7 y 8. Sólo los materiales arcillosos que han sido modificados (lixiviados) presentan un buen comportamiento en cuanto a adsorción de Cd, muy semejantes a los de la Vermiculita.

En el caso del cromo, el comportamiento ha sido a la inversa, las muestras sin modificar (ricas en geles de hierro y aluminio) son las que más $\mathrm{Cr}$ han retenido, debido probablemente a la formación de enlaces del tipo $\left(\mathrm{Cr}_{\mathrm{x}} \cdot \mathrm{Fe}_{1-x}\right)$ $(\mathrm{OH})_{3}$, siendo x la fracción molar de Cr (25-26).

\section{CONCLUSIONES}

Se han caracterizado térmica y tecnológicamente las muestras de minerales arcillosas del yacimiento Cayo Guan del municipio de Moa de Cuba para establecer su aplicación como materia prima en el campo cerámico.

- $\quad$ El índice de plasticidad de las muestras está comprendido entre 13 y 0,5\%. Sólo una de las muestras, CG-2, presenta buena plasticidad (13\%) para ser utilizada directamente en el procesamiento por el método de extrusión. El resto de las muestras son susceptibles de una fácil regulación.

- Los diagramas de gresificación, así como la microscopía de calefacción han permitido conocer la alta refractariedad de las muestras. A partir de $1300{ }^{\circ} \mathrm{C}$ es cuando aparecen signos de vitrificación, pero es a $1400{ }^{\circ} \mathrm{C}$ cuando las contracciones lineales son máximas y las absorciones de agua $<0,5 \%$. Esto concuerda con el alto contenido en alúmina y el bajo en óxidos alcalinos. 
- Empleando un programa de cálculo por ordenador basado en la herramienta Solver se ha diseñado y obtenido una amplia gama de materiales cerámicos con posibilidades, a falta de un estudio económico, de ser empleados como ladrillos cara vista o gres rojo. A título de ejemplo, el gres obtenido utilizando como materia prima $60 \%$ en peso de la arcilla CG-2, $30 \%$ de feldespato potásico, 10 $\%$ de arena y un tratamiento térmico a $1250{ }^{\circ} \mathrm{C} / 90 \mathrm{~min}$ presenta unas propiedades tecnológicas y mecánicas que se corresponden con las normalizadas para un material de este tipo.

- Las muestras presentan un buen comportamiento para ser utilizadas como adsorbentes de iones $\mathrm{Cr}$ debido a su alto contenido de oxi-hidróxidos de hierro y aluminio, y después de su activación ácida con $\mathrm{HCl}$ para los iones $\mathrm{Cd}$.

\section{AGRADECIMIENTOS}

Este trabajo ha sido parcialmente financiado por el MICINN - España a través del proyecto MAT 2007-62601

Los autores agradecen a la Agencia Española de Cooperación Internacional (AECI) la concesión de la beca para investigar en el ICV-CSIC durante dos años a Daris Fonseca.

\section{BIBLIOGRAFÍA}

1. A. Rojas, A. Turro; “Composición mineralógica de las colas del proceso Caron en Moa, Holguín, Cuba". Minería y Geología, 3-4 22-28 (2003)

2. J. L. Otero de la Gándara, P. Álvarez. "La magnetita en la fabricación de ladrillos para la acumulación del calor". Rev. Metal. Madrid, 30 [3] 135144 (1994)

3. V.I. Kharitonov "Radiation shielding bricks from metallurgical products", Industrial Ceramics, 22 [3] 169-174 (2002)

4. R.G. Sonkawade, K. Kant, S. Muralithar, R Kumar, R. C. Ramala. “Natural radioactivity in common building construction and radiation shielding materials". Atmospheric Enviroment 42 2254-2259 (2008)

5. D. Fonseca, F. Barba, P. Callejas, P. Recio. “Comportamiento térmico de los minerales no metálicos de Cayo Guan, Cuba" Boletín de la SECV, 48 [3] Mayo-Junio, pp 171- 178, (2009)

6. F. L. Marabih, G. Orozco, D. Fonseca. “Evaluación preliminar y caracterización de la manifestación de caolinitas en la zona de Cayo Guan, Moa". Informe Técnico. ISMM, Cuba (2005)

7. Norma española UNE 103-104-93: “Determinación del límite plástico de un suelo", febrero, 1993
8. Norma UNE 103-103-93: “Determinación del limite liquido de un suelo por el método del aparato de Casagrande", 1993

9. L. A. Díaz Rodríguez.; R. Torrecillas. "Arcillas wealdenses en Cantabria (N. de España): Su aprovechamiento cerámico". Boletín de la SECV Vol. 45 [1] 46-51 (2006)

10. Norma UNE 67027-84: Ladrillos de arcilla cocida. Determinación de la absorción de agua.

11. UNE-EN ISO 10545-8, 199: Determinación de coeficiente de dilatación lineal de materiales cerámicos-BALDOSAS; UNE -EN ISO 10545-8 1997 ERRATUM 2008

12. ASTM C1327-99 "Standart Test Method for Vickers Indentation Hardness of Advanced Ceramics Designation", (1999)

13. S. Tamborenea, A.D. Mazzoni, E.F. Aglietti. "Mechanochemical activation of minerals on the cordierite synthesis". Thermochimica Acta 411, 219-224 (2004)

14. U. Schwertmann, "The double dehydroxylation peak of goethite". Thermochimica Acta 78 (1984) 39-46.

15. C.Y. Chen, G.S. Lan, W.H. Tuan, Microstructural evolution of mullite during the sintering of kaolin powder compacts. Ceramics international, 26 (2000) 715-720.

16. J. Temuujin, K.J.D. MacKenzie, M. Schmucker, H. Schneider, J. McManus, S. Wimperis. Phase evolution in mechanically treated mixtures of kaolinite and alumina hydrates (gibbsite and boehmite). Journal of the European Ceramic Society 20 (2000) 413-421

17. W.E Lee, G.P Souza, C.J Mc Conville, "Mullite formation in clays and clay-derived vitreous ceramics" Journal of the European Ceramic Society 28 465-471 (2008)

18. M. Vázquez, J. Jiménez-Millán, C. Sánchez-Jiménez, J. Parras. "Composición y propiedades cerámicas de las pizarras de la zona centro ibérica del macizo ibérico meridional (Norte de Jaén, España)", 42 [4] Boletín Soc Esp Cer y Vid 215-221 (2003)

19. R. Galindo, "Pastas y Vidriados en la fabricación de pavimentos y revestimientos cerámicos" Colorobbia , España s.a, Faenza Editrice Ibérica s.l, España (1994)

20. F. Sandoval del Río, "Estructura cristalina de las arcillas y tecnología de su cocción". Curso de verano de El Escorial. Editorial Complutense s.a, España Nov. (1996)

21. L. Sanchez-Muñoz, J.B. Carda Castelló, "Materias primas y aditivos cerámicos". Enciclopedia cerámica II.2 Faenza Editrice Ibérica, s.l, Castellón España (2003)

22. J. F. Marciano M, A. Zanardo; J. M. Cabral. “As matérias-primas cerâmicas. Parte I: O perfil das principias indústrias cerâmicas e seus productos". Cerámica Industrial 6 [2] marzo-abril, (2001)

23. D. Walter, G. Buxbaum and W. Laqua, "The Mechanism Of The Thermal Transformation From Goethite To Hematite". Journal of Thermal Analysis and Calorimetry, 63 733-748, (2001)

24. A. Barba, V. Beltrán, y otros, "Materias primas para la fabricación de soportes de baldosas cerámicas". Instituto de Tecnología Cerámica. ITCAICE, ISBN 84-923176-0-4, Castañ S.L, Castellón, (España) (1997)

25. J. Kyziol-Komosinska; F. Barba; P Callejas, C. Rosik-Dulewska, "Bedeillite and other natural low-cost sorbents to remove chromium and cadmium from water and wastewater". 49 [2] Boletín Soc Esp Cer y Vid 121-128 (2010)

26. B.M. Sass; D. Rai. "Solubility of amorphous chromium(III) iron(III) hydroxide solid solutions" Inorg. Chem., 26. 2228-2232 (1987) 